\title{
Evaluation of Fractional Analysis of Bronchoalveolar Lavage Combined with Cellular Morphological Features
}

\author{
Namiko Taniuchi 1,2, Mohammad Ghazizadeh 1, Tatsuji Enomoto 3, Kiyoshi Matsuda 4, Masashi Sato 5, Yuko \\ Takizawa ${ }^{1}$, Enjing Jin 1, Seiko Egawa ${ }^{1}$, Arata Azuma ${ }^{2}$, Akihiko Gemma ${ }^{2}$, Shoji Kudoh ${ }^{2}$, Oichi Kawanami ${ }^{1}$ \\ $凶$
}

1. Department of Molecular Pathology, Institute of Development and Aging Sciences, Nippon Medical School, Graduate School of Medicine, Kawasaki, Japan.

2. Fourth Department of Internal Medicine, Nippon Medical School, Tokyo, Japan.

3. Department of Respiratory Medicine, Tokyo Metropolitan Hiroo Hospital, Tokyo, Japan.

4. Department of Emergency and Critical Care Medicine, Yamanashi Prefectural Central Hospital, Yamanashi, Japan.

5. Department of Radiology, Nippon Medical School Musashi-kosugi Hospital, Kawasaki, Japan.

$\triangle$ Correspondence to: Oichi Kawanami, MD, PhD, Professor and Chairman, Department of Molecular Pathology, Institute of Development and Aging Sciences, Nippon Medical School, Graduate School of Medicine, 1-396 Kosugi-cho, Nakahara-ku, Kawasaki, Japan 211-8533. Tel:81-44-733-1823; Fax:81-44-733-1293; kawanami@nms.ac.jp

Received: 2008.10.20; Accepted: 2008.11.26; Published: 2008.12.01

Background. The value of bronchoalveolar lavage (BAL) still remains controversial, prompting a need for further improvement. The purpose of this study was to develop and evaluate a sequential analysis of cell content in fractional BAL (FBAL) from the airways and alveolar sacs with incorporation of the cellular morphologic features. Methods. Initially, $30 \mathrm{ml}$ saline was infused into a subsegmental lobe of the lung and the recovered fluid was assigned as FBAL-I being mainly originated from whole airways. The second and third lavages (FBAL-II and FBAL-III) each were performed using $50 \mathrm{ml}$ saline being from more distal portions of airways and alveolar sacs respectively in the same lobe. Total cell number/ml and percentages of macrophages, lymphocytes, neutrophils, and eosinophils in each fraction together with their morphological alterations and mast cells, basophils and Masson bodies were assessed. Results. In the 12 controls, percentage of neutrophils was high and lymphocytes and macrophages were low in FBAL-I while in FBAL-III, neutrophils decreased to nearly nil and lymphocytes and macrophages were increased. Analysis of FBAL from 76 patients with sarcoidosis and 14 with hypersensitivity pneumonitis (HP) revealed that a predominance of small, round and well-differentiated lymphocytes with relative absence of neutrophils, basophils and Masson bodies correlated best with sarcoidosis. In contrast, neutrophil predominance and presence of lymphocytes having deep nuclear indentations and abundant cytoplasm with a process resembling a "hand-mirror" correlated well with HP. Conclusions. Evaluation of FBAL together with cellular morphological features especially characteristics of lymphocytes provides valuable information for establishing the diagnosis in interstitial lung diseases.

Key words: Bronchoalveolar lavage procedure; interstitial lung diseases; sarcoidosis; hypersensitivity pneumonia; lymphocyte morphology; neutrophil; Masson bodies.

\section{Introduction}

Bronchoalveolar lavage (BAL) has been widely applied as a routine tool in laboratories of respiratory disease ${ }^{1}$. The BAL technique is safe and minimally invasive, and the presence of cell patterns can support clinical diagnosis in the absence of biopsy or when the clinical feature is compatible 2,3 . However, the application of BAL still remains controversial as the results obtained so far were based on a variety of techniques or methods thus introducing considerable variations in the implications ${ }^{4}$. Although it is well recognized that correlations between BAL fluid cells and corresponding tissue cell extractions are better for lymphocyte infiltration in granulomatous lung disease 5 , morphological features of lymphocytes have not been well defined and incorporated in relation to the pathological conditions. 
The primary objective of this study was to develop a sequential analysis of cells in FBAL with special references to the distribution of inflammatory cells in the airways and alveolar sacs combined with morphological alteration of lymphocytes in relation to the functional states; migration of mast cells and basophils. Using this method, lymphocyte morphology in hypersensitivity pneumonitis (HP) was sharply contrasted with the other lymphocyte-rich diseases such as sarcoidosis.

\section{Materials and Methods}

Fractional analysis of cells in BAL fluid. After premedication with atropine and hydroxyzine, a bronchoscope was inserted under local anesthesia with lidocaine and wedged at orifice of the objective sub-segment of a lobe. The first lavage was done with a $30 \mathrm{ml}$ of sterile $0.9 \%$ saline (FBAL-I) which was assigned as "whole airway" lavage. Subsequently, the second (FBAL-II) and third (FBAL-III) lavages were performed each with a $50 \mathrm{ml}$ saline and were considered to be from "distal airway" and "alveolar sac" respectively (Figure 1). The percent recovery rate of lavage fluid and a total cell number were estimated in each FBAL, and then cytocentrifuging was done for 5 minutes at $1,500 \mathrm{rpm}$ at room temperature (Model Cytospin 3, Shandon Southern Products Ltd., UK) for microscopic evaluation. The cells were stained by May-Grunwald Giemsa and Papanicolaou methods. The differential cell counts were performed under a light microscope by counting more than 300 cells. Mast cells and basophils in FBAL were counted and graded as $0,1+\left(1 / 10^{3}\right.$ cells $), 2+\left(2 \sim 5 / 10^{3}\right.$ cells $)$ and $3+\left(>5 / 10^{3}\right.$ cells $)$. We further checked non-cellular components, including tiny organized proteinous accumulations or Masson bodies, foreign bodies, aggregates.

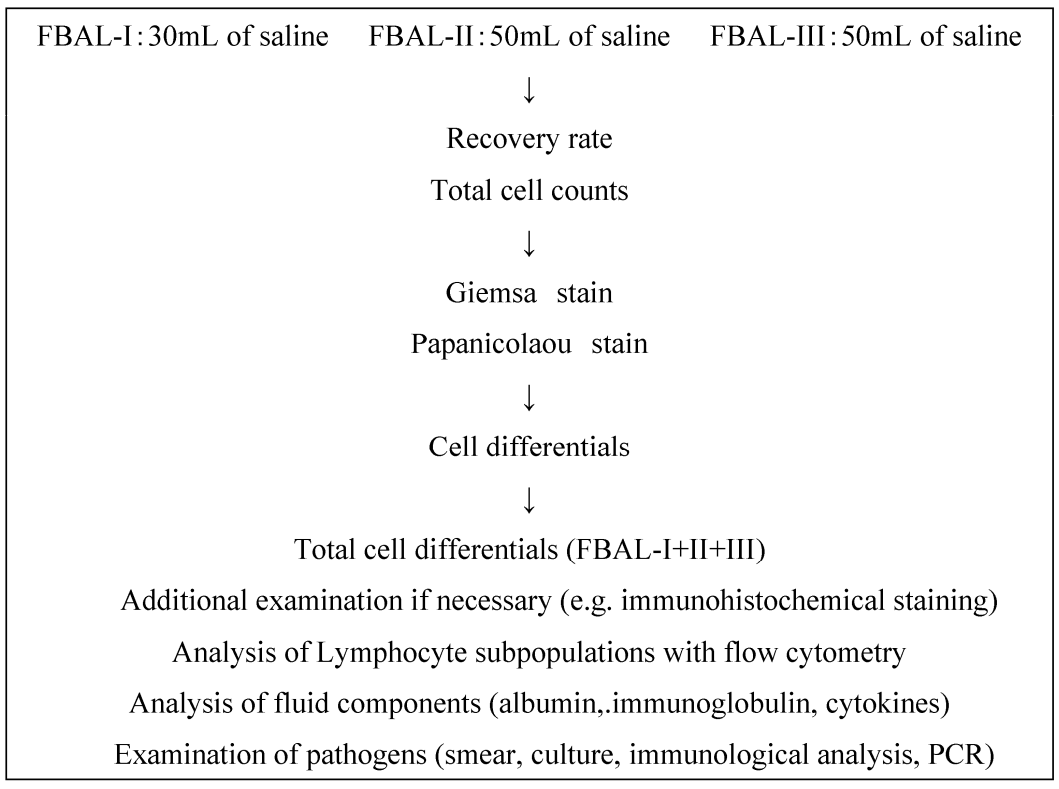

Figure I. Fractional analysis of bronchoalveolar lavage (FBAL) procedure. PCR: polymerase chain reaction.

Control individuals and patients with lung diseases. Control individuals were comprised 8 males (mean age \pm SD: $45.0 \pm 13.0$ ), and 4 females (44.8 \pm 17.7$)$. We reviewed clinicopathological records of a total of 2260 patients with various lung diseases between 1984 and 2006 at our Nippon Medical School affiliated hospitals (Table 1). Among them, 76 of sarcoidosis and 14 of HP patients had both a definite diagnosis with histopathological confirmation and all three FBAL samples available and therefore selected for detailed studies. Written informed consent was obtained from each patient when they underwent the examinations.
This study was also approved by the Ethical Committee of the Nippon Medical School.

Clinical features of patients with sarcoidosis. The 76 patients, including 39 men (35.8 \pm 14.5$)$ and 37 women (51.8 \pm 13.5$)$, were proved by lung biopsy to have developed characteristic non-caseating epithelioid granuloma. Based on chest radiographs, the patients were divided into four stages according to the criteria established by the American Thoracic Society, European Respiratory Society and WASOG ${ }^{6}$. Seven patients $(9.2 \%)$ met stage 0,39 patients $(51.3 \%)$ stage I, 14 patients (18.4\%) stage II, and 16 patients $(21.1 \%)$ 
stage III. Thirty-seven patients $(48.7 \%)$ showed multiple organ involvements of sarcoidosis including skin, eyes and/or heart. As to smoking habits, 31 $(40.8 \%)$ were current smokers, 37 patients $(48.7 \%)$ had never smoked or were past smokers.

Table I. Clinical Diagnoses (Nippon Medical School-affiliated hospitals, April 1984 - February 2006).

\begin{tabular}{|l|l|}
\hline Clinical diagnoses & Patients (\%) \\
\hline Interstitial lung diseases & $734(32.5)$ \\
\hline IPF/UIP & $101(4.5)$ \\
\hline NSIP & $33(1.5)$ \\
\hline COP/BOOP & $109(4.8)$ \\
\hline CVD-IP & $145(6.4)$ \\
\hline The others with IP & $346(15.3)$ \\
\hline Sarcoidosis & $265(11.7)$ \\
\hline Radiographic abnormal shadows & $136(6.0)$ \\
\hline Eosinophilic pneumonia & $119(5.3)$ \\
\hline Leukemia, lymphoma and others & $104(4.6)$ \\
\hline Hypersinsitivity pneumonitis & $104(4.6)$ \\
\hline Pneumoconiosis (Asbestosis, Coal workers) & $95(4.2)$ \\
\hline Malignant tumor (Primary or metastat. Cancer) & $81(3.4)$ \\
\hline Tuberculosis & $73(3.2)$ \\
\hline ARDS/AIP & $64(2.8)$ \\
\hline Fungus, P.Carinii & $42(1.9)$ \\
\hline Bloody sputum & $30(1.3)$ \\
\hline Langerhans cell histiocytosis & $19(0.8)$ \\
\hline Alveolar proteinosis & $12(0.5)$ \\
\hline Mycobacterium infection & $11(0.5)$ \\
\hline Wegener's granulomatosis & $4(0.2)$ \\
\hline The others & $367(16.2)$ \\
\hline Total & 2260 \\
\hline
\end{tabular}

IPF: idiopathic pulmonary fibrosis

UIP: usual interstitial pneumonia

NSIP: non-specific interstitial pneumonia

COP: cryptogenic organizing pneumonia

BOOP: bronchiolitis obliterans organizing pneumonia

CVD-IP: collagen vascular disease-related interstitial pneumonia

ARDS: acute respiratory distress syndrome

AIP: acute interstitial pnemonia

Clinical features of hypersensitivity pneumonitis. The 14 patients, including 6 men $(65.3 \pm 7.1)$ and 8 women (59.0 \pm 9.4$)$, were proved by lung biopsy to have developed HP. Six patients were non-smokers, and 8 current smokers. Four patients were diagnosed as having summer type hypersensitivity pneumonitis $^{7}, 4$ patients had hypersensitivity to chemical material, 1 to feathers of bedding, and 5 to unknown house dust antigens. In these 5 patients, typical symptoms were provoked, and besides, laboratory data were compatible when they returned home or to work place after cessation of symptoms during brief hospitalization. Biopsied lung tissues were compatible with HP, including diffuse infiltration of lym- phocytes, intra-alveolar corporation of collagenous exudate (Masson body) and tiny non-caseating epithelioid granuloma formation.

Statistical Analysis. Paired-sample $t$-test was used to determine whether there was a statistically significant difference between each cell differentials of patients with sarcoidosis or HP (SPSS Software, SPSS Japan Inc.). We focused on the relationship between the fractions, including FBAL-I, FBAL-III and FBAL-total, radiographic staging and smoking history. A $p$-value $<0.05$ was considered statistically significant.

\section{Results}

Cell differential rates in control individuals. Among control group, neutrophil percentage was highest in FBAL-I and decreased to nearly nil in FBAL-III $(p<0.05)$. In contrast, lymphocytes and macrophages were slightly increased in FBAL-III. The differential cell rates in each FBAL did not show any significant differences related to the smoking habit (Table 2).

Table 2. Cell differential ratios in control individuals $(n=12)$ and when subdivided into smokers and non-smokers.

\begin{tabular}{|c|c|c|c|c|c|}
\hline \multicolumn{6}{|c|}{ Control individuals $(n=12)$} \\
\hline & $\begin{array}{l}\text { Cell number } \\
\left(\times 10^{5} / \mathrm{mL}\right)\end{array}$ & $\mathrm{M} \varphi(\%)$ & $\operatorname{Ly}(\%)$ & Neu(\%) & $\operatorname{Eo}(\%)$ \\
\hline FBAL-I & $1.08 \pm 0.45$ & $88.5 \pm 3.0$ & $5.8 \pm 1.2$ & $4.7 \pm 1.9 *$ & $1.0 \pm 0.45$ \\
\hline FBAL-II & $1.83 \pm 0.63$ & $90.5 \pm 1.1$ & $7.5 \pm 0.99$ & $1.5 \pm 0.50$ & $0.33 \pm 0.21$ \\
\hline FBAL-III & $2.52 \pm 0.76$ & $91.7 \pm 1.2$ & $7.3 \pm 0.84$ & $0.33 \pm 0.21$ & $0.33 \pm 0.21$ \\
\hline FBAL-total & $1.84 \pm 0.47$ & $91.0 \pm 1.2$ & $7.0 \pm 0.73$ & $1.3 \pm 0.33 *$ & $0.50 \pm 0.22$ \\
\hline \multicolumn{6}{|c|}{ Control smokers $(n=6)$} \\
\hline & $\begin{array}{l}\text { Cell number } \\
\left(\times 10^{5} / \mathrm{mL}\right)\end{array}$ & $\mathrm{M} \varphi(\%)$ & $\operatorname{Ly}(\%)$ & Neu(\%) & $\mathrm{Eo}(\%)$ \\
\hline FBAL-I & $1.4 \pm 1.07$ & $85.0 \pm 4.6$ & $7.7 \pm 0.88$ & $6.0 \pm 3.5$ & $1.3 \pm 0.67$ \\
\hline FBAL-II & $3.01 \pm 1.39$ & $89.0 \pm 1.2$ & $8.3 \pm 1.9$ & $2.3 \pm 0.67$ & $0.33 \pm 0.33$ \\
\hline FBAL-III & $3.08 \pm 1.04$ & $90.3 \pm 1.2$ & $8.7 \pm 0.67$ & $0.67 \pm 0.33$ & $0.33 \pm 0.33$ \\
\hline FBAL-total & $2.45 \pm 0.78$ & $89.7 \pm 1.2$ & $8.0 \pm 0.58$ & $1.7 \pm 0.33$ & $0.67 \pm 0.33$ \\
\hline \multicolumn{6}{|c|}{ Control nonsmkers $(n=6)$} \\
\hline & $\begin{array}{l}\text { Cell number } \\
\left(x 10^{5} / \mathrm{mL}\right)\end{array}$ & $\mathrm{M} \varphi(\%)$ & $\operatorname{Ly}(\%)$ & Neu(\%) & $\mathrm{Eo}(\%)$ \\
\hline FBAL-I & $0.87 \pm 0.35$ & $92.0 \pm 3.2$ & $4.0 \pm 1.5$ & $3.3 \pm 1.9$ & $0.67 \pm 0.67$ \\
\hline FBAL-II & $1.04 \pm 0.33$ & $92.0 \pm 1.5$ & $6.7 \pm 0.88$ & $0.67 \pm 0.33$ & $0.33 \pm 0.33$ \\
\hline FBAL-III & $1.96 \pm 1.18$ & $93.0 \pm 2.1$ & $6.0 \pm 1.2$ & $0.0 \pm 0.0$ & $0.33 \pm 0.33$ \\
\hline FBAL-total & $1.22 \pm 0.47$ & $92.3 \pm 2.0$ & $6.0 \pm 1.2$ & $1.0 \pm 0.58$ & $0.33 \pm 0.33$ \\
\hline
\end{tabular}

${ }^{*} \mathrm{p}<0.05$ as compared with \% neutrophils in FBAL-III.

M $\phi$ : macrophages, Ly: lymphocytes, Neu: neutrophils, Eo: eosinophils

Patients' group showing the highest rate of lymphocytes. Of the 2260 patients, $488(21.6 \%)$ showed a lymphocyte rate of higher than $40 \%$. Clinical diagnoses of these patients included sarcoidosis (22.1\%), IIP (14.5\%), HP (11.5\%), leukemia/lymphoma including tumor cells $(10.9 \%), \mathrm{COP} / \mathrm{BOOP}(9.0 \%)$ and others (Table 3). Sarcoidosis and HP are the most distinguished group of diseases with lymphocyte-related 
etiology and the diagnosis of these granulomatous diseases were well defined by lung biopsy.

Table 3. Patients' group showing a highest ratio of lymphocyte in FBAL-total

\begin{tabular}{ll}
\hline Clinical Diagnosis & Patients (\%) \\
Sarcoidosis & $108(22.1)$ \\
IIPs & $72(14.5)$ \\
Hypersensitivity Pneumonitis & $38(11.5)$ \\
Leukemia/Lymphoma & $53(10.9)$ \\
COP/BOOP & $44(9.0)$ \\
Tuberculosis & $18(3.7)$ \\
Lung cancer & $10(2.0)$ \\
Others & $145(29.7)$ \\
Lymphocytes $>40 \%$ in FBAL-total & 488 \\
\hline
\end{tabular}

IIPs: idiopathic interstitial pneumonias

COP: cryptogenic organizing pneumonia

BOOP: bronchiolitis obliterans organizing pneumoni

Differential cell rates in sarcoidosis. The mean cell numbers in 76 sarcoidosis patients were $1.64 \pm 0.30$ $x 10^{5} / \mathrm{mL}$ in FBAL-I, $3.26 \pm 0.38 \times 10^{5} / \mathrm{mL}$ in FBAL-III, and $2.96 \pm 0.36 \times 10^{5} / \mathrm{mL}$ in FBAL-total (1.6-fold increase vs. control). The higher percentages of lymphocytes in each fraction were $40.8 \pm 2.2 \%(3.9-82.8 \%)$ in FBAL-total, $28.5 \pm 2.2 \%$ in FBAL-I, and $43.9 \pm 2.3 \%$ in FBAL-III respectively (Figure 2). The mean lymphocyte percentage of patients who had lung involvement (stage II or III) did not differ in clinical symptoms and laboratory data from those of patients with no lung involvement (stage 0 or I) $(40.3 \pm 3.7 \%$ vs. $41.2 \pm 2.7 \%$ ). There were 40 patients, including 18 smokers, who showed no neutrophil recovery in any fractions. Among sarcoidosis smoker patients $(n=31)$, mean neutrophil rate was $4.6 \pm 2.1 \%$ in FBAL-I compared to $0.8 \pm 0.2 \%$ in FBAL-III $(p=0.06)$. On the other hand, among sarcoidosis nonsmokers $(n=37)$, mean neutrophil rate was $9.7 \pm 3.4 \%$ in FBAL-I compared to $3.2 \pm 1.2 \%$ in FBAL-III $(p<0.05)$.

Differential cell rates in hypersensitivity pneumonitis. The average total cell number was $1.59 \pm 0.33$ $\mathrm{x} 10^{5} / \mathrm{mL}$ in FBAL-I, $6.45 \pm 0.92 \times 10^{5} / \mathrm{mL}$ in FBAL-III, and $5.29 \pm 0.70 \times 10^{5} / \mathrm{mL}$ in FBAL-total showing a 2.9-fold increase versus normal control $(p<0.05)$ and 1.8 -fold higher than sarcoidosis $(p<0.05)$. A significantly higher proportion of lymphocytes was seen (mean $65.2 \pm 4.1 \%$ ranging from 35.9 to $84.3 \%$ ) and the difference of lymphocyte rates between FBAL-I $(47.5 \pm 5.0 \%)$ and FBAL-III (68.9 $\pm 4.5 \%)$ was statistically significant. Neutrophil rate in FBAL-I was $17.4 \pm 3.4 \%$ in contrast to $2.9 \pm 0.7 \%$ in FBAL-III $(p<0.05)$ and $4.4 \pm 0.9 \%$ in FBAL-total among HP patients, which was significantly higher than in the normal control
$(1.3 \pm 0.33 \% ; P<0.05)$ (Figure 3). Lymphocyte rate in FBAL-total in HP smoking patients tended to be lower than non-smoking patients $(60.6 \pm 4.9 \%$ vs. $68.1 \pm 9.7 \%)$, and neutrophil rate in FBAL-total showed a higher level in nonsmokers than in smokers ( $5.5 \pm 1.9 \%$ vs. $3.6 \pm 1.2 \%$; no statistical significance).

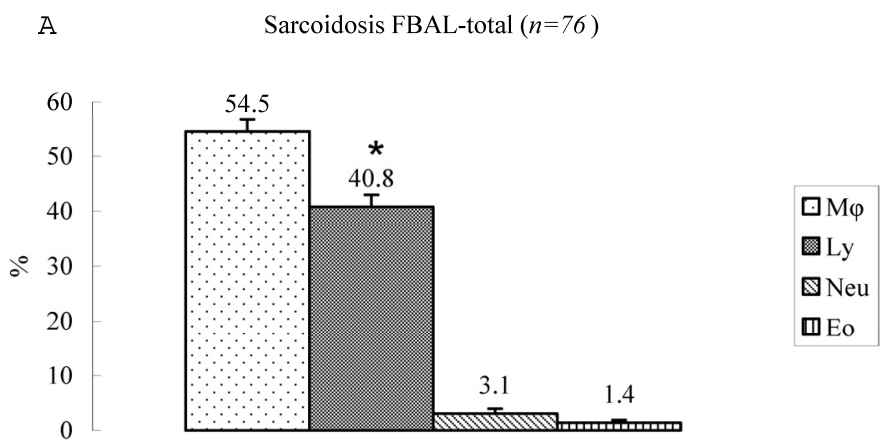

B Sarcoidosis FBAL-I $(n=76)$

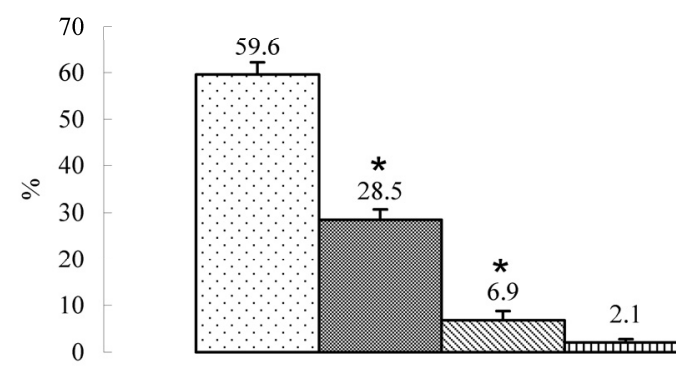

$\square \mathrm{M \varphi}$ Ly SNeu पEo

C Sarcoisosis FBAL-III $(n=76)$

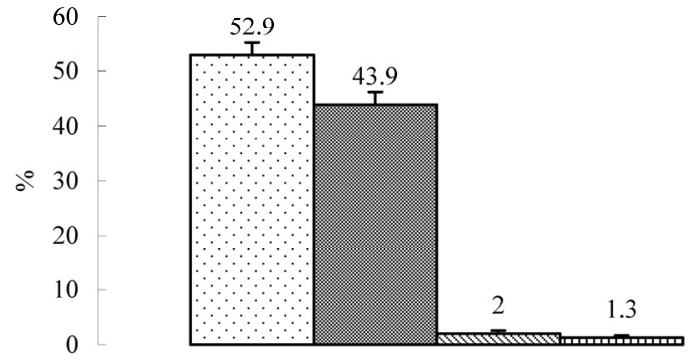

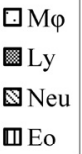

Figure 2. Cell differentials in sarcoidosis $(n=76)$. BAL fluid cells in sarcoidosis patients were characterized by a higher percentage of lymphocyte at each fraction, showing $40.8 \pm 2.2 \%$ (3.9-82.8\%) FBAL-total (A), while that in FBAL-I (B) was $28.5 \pm 2.2 \%$ compared to $43.9 \pm 2.3 \%$ in FBAL-III (C) $(p<0.05)$. Mean neutrophil rate was $3.1 \pm 0.9 \%$ in FBAL-total among all patients with sarcoidosis, while mean neutrophil rate in FBAL-I was $6.9 \pm 1.9 \%$ compared to $2.0 \pm 0.6 \%$ in FBAL-III $(p<0.05)$. Asterisk indicates a significant difference from FBAL-III $(p<0.05)$. 

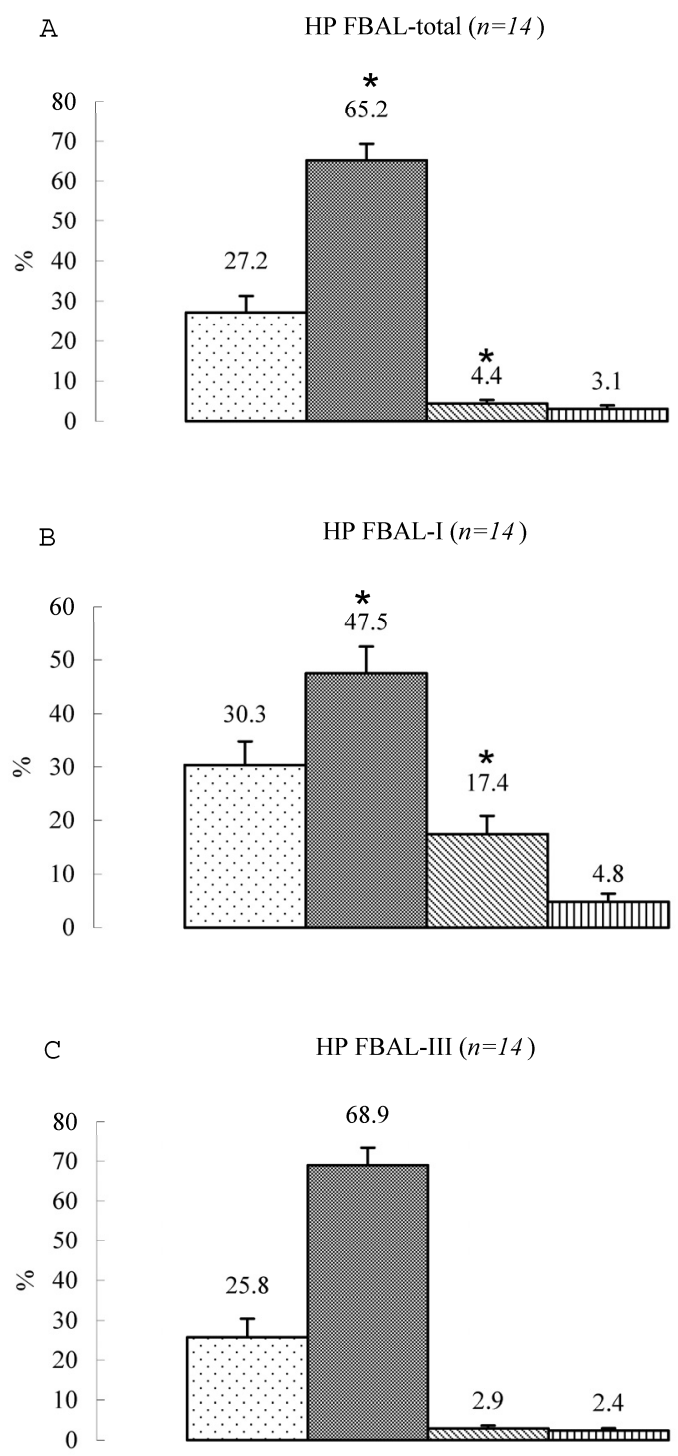

Figure 3. Cell differentials in hypersensitivity pneumonitis $(n=14)$. A significantly higher proportion of lymphocyte was seen in HP patients (mean $65.2 \pm 4.1 \%$ ranging from 35.9 to $84.3 \%$ ). The difference of lymphocyte rates between FBAL-I (A; $47.5 \pm 5.0 \%$ ) and FBAL-III (C; 68.9 $\pm 4.5 \%$ ) was statistically significant $(p<0.05)$. Neutrophil rate in FBAL-I was $17.4 \pm 3.4 \%$ in contrast to $2.9 \pm 0.7 \%$ in FBAL-III $(p<0.05)$ and $4.4 \pm 0.9 \%$ in FBAL-total $(A)$ among HP patients, which was significantly higher than in the normal control $(1.3 \pm 0.33 \%)(P<0.05)$. Asterisk indicates a significant difference from FBAL-III $(p<0.05)$.

Comparison of lymphocyte morphology between sarcoidosis and HP. Sarcoid-lymphocytes were generally small in size and appeared mature and well differentiated in morphology (Figure 4). They had a round nucleus and the cytoplasm was fairly thin. In contrast, HP lymphocytes had fairly abundant cytoplasm and occasionally developed an elongated cytoplasmic process resembling a hand-mirror in shape. A num- ber of lymphocytes looked like plasma cell, having large basophilic cytoplasm and nucleus with wheel-like appearance. In others, the nuclear membrane was highly convoluted like cerebelli-form or clover-shape. Nucleus often contained a large nucleolus. Sarcoidosis patients consistently showed a different morphology compared with HP patients. Other inflammatory cells, particularly eosinophils and mast cells were also frequently seen in BALF of HP patients (Figure 5).

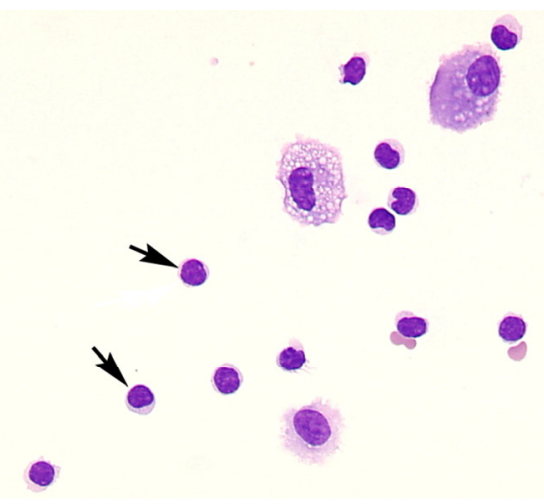

Figure 4. Morphology of sarcoid lymphocytes. The sarcoid lymphocytes displayed mature, small shape dominant, and also regular size cells. They had round-shape nuclei and scanty cytoplasm. (Giemsa stain $x$ original magnification $\times 200$ ).

Eosinophils, mast cells and basophils in FBAL. Higher eosinophil recovery $(>5 \%)$ in FBAL-total was found in 120 of 546 (22\%) of biopsy proven cases. The distribution of diseases were as follows; IIP 43 (35.8\%), eosinophilic pneumonia $18(15.0 \%)$, sarcoidosis $6(5.0 \%)$, drug-induced pneumonia $3(2.5 \%)$ and others. On the other hand, eosinophil recovery rate was highest in the group of eosinophilic pneumonia showing $28.2 \%$ following by NSIP $6.1 \%$, IPF $5.0 \%$ and COP/BOOP $4.6 \%$. With May-Grunwald-Giemsa staining, mast cells were identifiable by the purple-red granules in cytoplasm, and were encountered in half of a total 546 biopsy cases. Highest level of mast cells $(3+)$ were encountered in 96 patients with a variety of interstitial lung diseases, including 37 patients with IIP, 10 sarcoidosis, 7 eosinophilic pneumonia, and others. Distribution of mast cells in $7 \mathrm{HP}$ patients examined in this study were $3+(n=3), 2+$ $(n=3)$ and 1/0 (n=1). Basophilic leukocytes were never found in the normal control and most patients. Basophils were occasionally found in 13 patients with IIP, 3 HP, 5 eosinophilic pneumonia, and others. The three HP patients who contained basophils were all at 
relatively acute stage of HP symptoms and had recently received anti-flu or antibiotics for common cold or with tentative diagnosis of bacterial pneumonia. Acute eosinophilic pneumonia also showed basophil recovery as well as mast cells.

Masson body in the FBAL from HP patients. Formation of intra-alveolar budding or traditionally called "Masson body" was found in 8 patients; one each with HP, IPF, NSIP, IIPs, dermatomyositis, ARDS, organized hemosiderosis and adenocarci- noma. The existence of such organization lesion is considered one of the major histological characteristics including marked lymphocytic infiltration and formation of tiny granulation tissues with epithelial cell coverage in small airways and alveolar zones. Masson bodies in BALF torn off from HP alveolar walls consisted of the central collagen or fibrin part (red color expression by Papanicolaou stain) covered with regenerative epithelial cell lining (Figure 6). HP macrophages often had a foamy appearance.
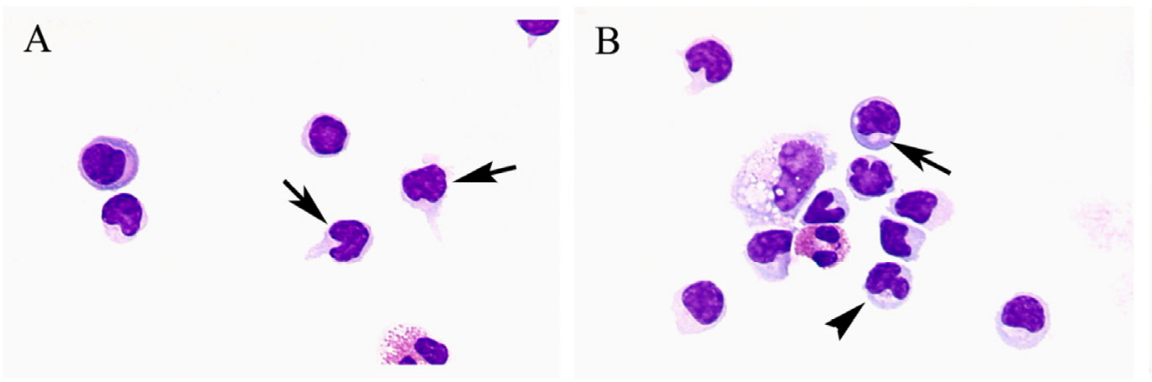

C

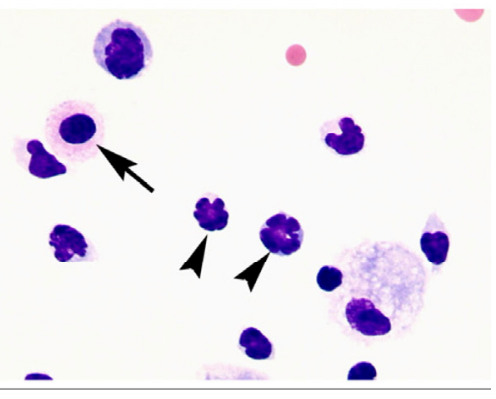

Figure 5. Morphology of hypersensitivity pneumonitis (HP) lymphocytes. HP lymphocytes morphology and diameter varied considerably. The cell morphology was consistent with activated-type lymphocyte. They presented cytoplasmic formations like hand-mirror cells which had cytoplasmic tail (A, arrows), or plasmacytoid cells which had light cytoplasmic regions adjacent to nuclei (B, arrow). They also had irregularities in the contours of the nuclear membranes such as cerebelli-form (B and C, arrowheads), glove mitt-like or clover-shaped. Other inflammatory cells, particularly eosinophils and mast cells (C, arrow) were also frequently seen in BALF of HP patients. (Giemsa stain; original magnification $\times 400$ ).

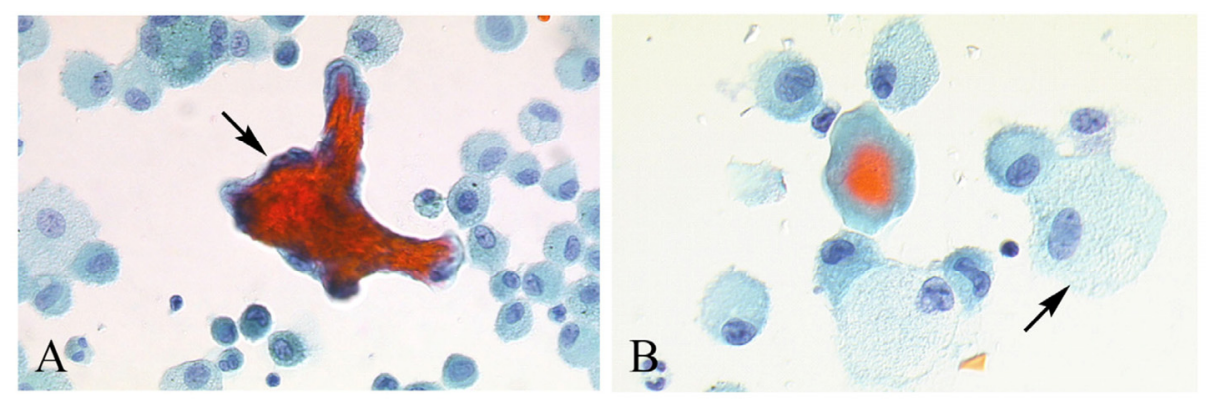

Figure 6. Masson body in BALF from HP patients. Masson bodies (intraalveolar foci of organizing pneumonia) in BALF are torn off from HP alveolar walls, although the frequency is low. Masson bodies ( $\mathbf{A}$, arrow) which are synonymous with collagen globules, are constructed of the central collagen or fibrin part (red color expression) covered with regenerative epithelial cell lining. There are also plenty of foamy macrophages (B, arrow). (Papanicolaou stain; original magnification x400).

\section{Discussion}

Our study aimed at evaluating a method of FBAL for determining cell distribution in airways and alveolar sacs separately in conjunction with cellular morphological features. In the control individuals, a higher neutrophil rate in FBAL-I indicated that constant exposure to outside air might evoke neutrophil accumulation on the surface of airway mucosa, which 
is in accord with the results shown by Yasuoka et al. (1985) ${ }^{8,9}$.

Eosinophils, mast cells and basophils were occasionally found among allergy-related patients including HP. The increased infiltration of mast cells implies histological feature of organization of collagenous matrices or interstitial fibrosis ${ }^{10}$. Basophilic leukocytes were never found in the normal control and most of the patients. The appearance of basophils was related to acute exacerbation of allergic disease especially in drug-induced pneumonia. Basophils have several morphological and functional characteristics in common with tissue mast cells, such as expression of the high-affinity IgE receptor which triggers a cascade of intracellular signals that lead to degranulation and synthesis of various mediators involved in the development and maintenance of allergic and inflammatory symptoms. Mast cells and basophils also display a number of growth-factor receptors and chemokine receptors, besides some of which are shared with eosinophils and account for the strong, temporal association of their influx into tissues ${ }^{11}$.

An intriguing point in our study was the cytological finding of Masson bodies. We found that Masson bodies can be occasionally seen in FBAL from $\mathrm{HP}$ alveolar walls, as they can easily desquamate from tissue surface into airspace. Masson bodies have been described in diffuse alveolar damage and were considered as the cytological counterpart of hyaline membrane and type II reactive cells ${ }^{12-14}$. However, the possibilities remain that this material may be the cytological counterpart of intra-alveolar loose fibrotic buds. The existence of such organized tissue fragments is an excellent hallmark of inflammatory lung tissue that triggers their development.

Among sarcoidosis patients in our study, mean neutrophil rate was increased in FBAL-I compared to FBAL-III, particularly in nonsmokers. Furthermore 18 smokers had no neutrophil recovery at any FBAL specimen. This result might suggest that neutrophils are not a major contributor to facilitate a milieu for lung sarcoidosis. Neutrophil infiltration often found in smokers' BAL fluid is rather limited in sarcoidosis, although the underlying reason awaits future clarification.

In summary, we developed and evaluated a sequential analysis of cell content in FBAL in patients with various interstitial lung diseases. This method determines inflammatory cell differentials in the airways and alveolar sacs separately together with assessment of the cellular morphological features. Our results showed that neutrophils consistently recovered from the airways surface regardless of smoking history. Lymphocytes were quite unusual in morphology, implying a functional activation in HP while lymphocyte rate in FBAL did not depend on activity of disease in sarcoidosis or HP. In addition, finding of encapsulated exudates may suggest desquamation of early stage of Masson body which implies possible diagnosis in a limited number of diseases. We believe that evaluation of FBAL together with cellular morphological features especially the characteristics of lymphocytes provides valuable information for establishing the diagnosis of specific interstitial lung disease.

\section{Conflict of Interest}

The authors have declared that no conflict of interest exists.

\section{References}

1. Reynolds HY, Newball HH. Analysis of proteins and respiratory cells obtained from human lungs by bronchial lavage. J Lab Clin Med 1974;84: 559-573

2. Hunninghake GW, Gadek JE, Kawanami O, Ferrans VJ, Crystal RG. Inflammatory and immune processes in the human lung in health and disease: evaluation by bronchoalveolar lavage. Am J Pathol 1979;97:149-206

3. Rottoli P, Bargagli E. Is bronchoalveolar lavage obsolete in the diagnosis of interstitial lung disease? Curr Opin Pulm Med 2004;10:320

4. Haslam PL, Baughman RP. Report of ERS Task Force: guidelines for measurement of acellular components and standardization of BAL. Eur Respir J 1999;14:245-248

5. Reynolds HY. Use of bronchoalveolar lavage in humans--past necessity and future imperative. Lung 2000;178:271-293

6. Statement on sarcoidosis. Joint Statement of the American Thoracic Society (ATS), the European Respiratory Society (ERS) and the World Association of Sarcoidosis and Other Granulomatous Disorders (WASOG) adopted by the ATS Board of Directors and by the ERS Executive Committee. Am J Respir Crit Care Med 1999;160:736-755

7. Kawai T, Tamura M, Murao M. Summer-type hypersensitivity pneumonitis. A unique disease in Japan. Chest 1984;85:311-317

8. Yasuoka S, Nakayama T, Kawano T, et al. Comparison of cell profiles of bronchial and bronchoalveolar lavage fluids between normal subjects and patients with idiopathic pulmonary fibrosis. Tohoku J Exp Med 1985;146:33-45

9. Ando M, Konishi K, Yoneda R, et al. Difference in the phenotypes of bronchoalveolar lavage lymphocytes in patients with summer-type hypersensitivity pneumonitis, farmer's lung, ventilation pneumonitis, and bird fancier's lung: report of a nationwide epidemiologic study in Japan. J Allergy Clin Immunol 1991;87:1002-100

10. Kawanami O, Ferrans VJ, Fulmer JD, et al. Ultrastructure of pulmonary mast cells in patients with fibrotic lung disorders. Lab Invest 1979;40:717-734

11. Arock M, Schneider E, Boissan M, et al. Differentiation of human basophils: an overview of recent advances and pending questions. J Leukocyte Biology 2002;71:557-564

12. Ambrosini V, Cancellieri A, Chilosi M, Zompatori M, Trisolini $\mathrm{R}$, Saragoni L, Poletti V. Acute exacerbation od idiopathic pulmonary fibrosis: report of aseries. Eur Resprir J 2003; 22:821-826 
13. Yoshinouchi T, Ohtsuki Y, Shikata Y. Clinicopathological study on two types of cryptogenic organizing pneumonitis. Resp Med 1995; 89:271-278

14. Dina R, Sheppard MN. The histological diagnosis of clinically documented cases of cryptogenic organizing pneumonia: diagnostic features in transbronchial biopsies. Histopathology 1993; 23:541-545 